\title{
A Joint Workshop of QACOS and OSSPL
}

\author{
Muhammad Ali Babar ${ }^{1}$, Björn Lundell ${ }^{2}$, and Frank van der Linden ${ }^{3}$ \\ ${ }^{1}$ Lero, Ireland \\ ${ }^{2}$ University of Skövde, Sweden \\ ${ }^{3}$ Philips Healthcare \\ malibaba@lero.ie, bjorn.lundell@his.se, \\ frank.van.der.linden@philips.com
}

\section{Introduction}

The OSS movement, which originated from a pragmatic need to share code among individuals, has grown to become a major force behind inter-organizational reuse of platforms, components and code. The use of open source software to build single or family of systems (i.e., product line development) appears to be a profitable way to quality software products. On the other hand, because of the diverse use of open source software, product line development is an attractive way of working in open source communities. The configuration mechanisms used in open source communities may be applicable within software product lines variability management. In addition, product line organizations are usually involved in distributed development, which works very efficiently within open source communities, leading to high quality products. However, at present, there is limited interaction between the open source and product line development communities.

There are several questions related to the quality and architectural aspects of the of open source software that need to be answered. The aim for the workshop is to explore what different communities (i.e., software architecture, software quality, software product lines, and open source software) whose work is closely related can learn from each other and to develop a better understanding of how these communities can benefit from each other. The workshop deals with the following issues:

1. The challenges of quality assurance of open source components

2. The architectural approaches used in OSS components and their support for seamless integration of OSS components in new systems and product lines.

3. The strengths and limitations of quality assurance and quality assessment approaches being used for selecting open source components

4. How to improve architecture and quality information exchange between open source communities and OS component users?

5. Community: Ownership, control and management of product line assets in an open source community

6. Visibility of the code: when it is valuable to share proprietary code and how to take the right decision.

7. Architecture Views: Creation of different levels of architecture visibility: proprietary, among closed consortium, public.

8. Model driven and service oriented approaches is open source communities 
9. The role of (open) standards in the integration of open source in product lines

10. Product line requirements, roadmaps and planning in open source development

11. Variability management: Using the open source community to evolve components and being explicit about variability

12. Variability representation, management and tooling in OSS community

13. Deployment: Open source for the platform and in applications

14. Tools: Open source asset management tools in product line development

15. The meaning of domain and application engineering in an open source context

16. Recovery and recognition of a product line in an open source asset base

17. Architectural measures for dealing with heterogeneous license requirements

18. Legal aspects dealing with evolutionary, variability or distribution of development relating to legal risks involving: liability, warranties, patent infringements etc.

\section{Workshop Goals}

The workshop aims at bringing together both researchers and practitioners who are interested in the quality and architectural issues in open source software and their use in software product lines. This workshop will be a joint gathering of the communities set up around two workshops, Quality and Architectural Concerns in Open Source Software (QACOS) and Open Source Software and Product Lines (OSSPL). The attendees of this workshop will discuss the importance and challenges of quality assurance, quality assessment, and architectural concerns involved in selecting and using open source components for developing and evolving large scale software intensive individual or family of systems.

The overall goal of the workshop is to gather several communities (i.e., software architecture, software quality, software product lines, and open source software) whose work is closely related to develop a common research agenda based on inputs from three communities to identify the research issues that should be addressed in the short to long term to establish relationships for cross-fertilization of research ideas and approaches. This event will also provide a platform to present mature and ongoing research aimed at:

- Bridging the gap between practitioners (open sources and commercial) and researchers on the selection, use and/or integration of open source components into in-house software for building product lines;

- Combining the advantages of open source, software quality, architecture-centric approaches, and software product lines. 